\title{
Treatment of diabetes mellitus
}

\author{
Work group chairman: R. P. Robertson ${ }^{1}$ \\ Report prepared by: D. J. Klein ${ }^{2}$ \\ ${ }^{1}$ Diabetes Center and ${ }^{2}$ Department of Pediatrics, University of Minnesota, Minneapolis, Minnesota, USA
}

Summary. New treatment strategies for subjects with Type 1 (insulin-dependent) and Type 2 (non-insulindependent) diabetes mellitus are being developed. Pilot studies utilising insulin itself have been reported to prevent Type 1 diabetes in subjects likely, by immunogenetic and physiologic criteria, to develop clinically overt disease, while the results of nicoti-namide trials in these subjects remain preliminary. Immunotherapy with cyclosporin $\mathrm{A}$ and azathioprine can slow disease progression and may produce long-term remissions when given within two months of onset of clinically overt disease.

In subjects with established disease, familial clustering of diabetic nephropathy may be related to concomitant susceptibility to hypertension and elevated rates of $\mathrm{Na} / \mathrm{H}$ countertransport. Treatment of hypertension associated with the nephropathy appears to slow renal deterioration. Whether reversal of the metabolic consequences of insulin deficiency or resistance also prevents chronic diabetic complications has not been firmly established. In the presence of a reduced Beta-cell mass, moderate hyperglycaemia may itself contribute to decreased muscle glucose uptake but not glycogen synthesis in Type 1 and Type 2 diabetes. Reversal of chronic hyperglycaemia by pancreas and islet cell transplantation, vanadate, and sulphonylureas are discussed as alternate strategies to insulin treatment in establishing normoglycaemia and furthering our understanding of insulin action and secretion at the cellular level. There remains a need to develop more sensitive biochemical and genetic markers to identify subjects at increased risk for developing chronic diabetic complications.

Key words: Diabetes mellitus, diabetic nephropathy, immuno-genetics, immuno-intervention, sulphonylurea, glucose, glucose transport, blood glucose control, transplantation.

\section{Introduction}

The purpose of this work group was to outline the state of the art regarding several modes of therapy currently being investigated for the prevention or treatment of Type 1 (insulin-dependent) and Type 2 (non-insulin-dependent) diabetes mellitus. The persistent metabolic morbidity as well as excess mortality related to chronic microvascular and macrovascular complications despite conventional therapy has prompted ongoing research into alternative strategies for the treatment of diabetes. For Type 1 diabetes, these therapeutic modalities can be divided into those implemented prior to the emergence of symptomatology, those which are instituted in an attempt to reverse the early metabolic alterations of the disease while endogenous Beta-cell function is still present, and those which are designed to delay the progression of early chronic diabetic complications in patients with absent endogenous insulin secretion. Studying the efficacy of proposed modalities in the "pre-diabetic" and overt diabetic states has generated new information concerning the natural history and pathophysiology of diabetes. This research has also furthered our understanding of the impact of environmental and secondary genetic factors on the primary determinants of susceptibility to the disease and its chronic complications.

\section{Prevention trials in Type 1 diabetes}

Eisenbarth reported that amongst first degree relatives of patients with Type 1 diabetes, approximately $1 / 50$ have high titre (greater than 20 JDF units) antibodies against islet cell cytoplasmic antigens (ICA) [1]. The presence of these circulating antibodies is associated with an increased risk for the development of overt diabetes, especially when associated with insulin autoantibodies. The disease may not become clinically apparent for months or even years after the detection of significant antibody titres. Standardisation 
of the ICA assay has shown that the absolute antibody titre may be related to the rate of progression towards overt disease.

One-third of relatives with positive ICA have other markers which may predict the future development of diabetes, such as high levels of insulin autoantibodies or depletion of first phase insulin secretion in response to an intravenous glucose tolerance test (IVGTT) [2]. A linear regression model has been developed using ICA titres and insulin responses by IVGTT which in selected individuals was found to predict with $90 \%$ confidence the development of overt diabetes within 4 years. Screening for antibodies to the $64 \mathrm{kDa}$ islet protein (glutamic acid decarboxylase or GAD) may play an important role in the detection of subjects at increased risk for Type 1 diabetes in the future [3].

Insulin administration to the BB Wistar rat or the NOD mouse prevented the development of insulitis and overt diabetes, as well as the ability to transfer the disease with lymphocytes while preserving C-peptide secretion [4]. Therefore, a preliminary trial was carried out in five prediabetic humans whose predicted time to onset of diabetes was less than 2.5 years by the linear regression equation described above and whose oral glucose tolerance tests were unimpaired. These patients were given regular insulin intravenously every 9 months and twice daily subcutaneous ultralente insulin $(<0.2 \mathrm{U} / \mathrm{kg})$. None developed diabetes after treatment for up to 3 years. In contrast, six of seven patients who declined treatment have developed overt disease during the ensuing 27 months. These encouraging preliminary results have led to a proposal for a larger, randomised trial to confirm the efficacy of insulin in halting the progression of pre-diabetes and to evaluate whether intravenously or subcutaneously administered insulin or both are required.

A large trial of nicotinamide is also planned for ICA positive relatives of patients with diabetes. Conflicting data concerning the efficacy of this agent have been presented, perhaps related to the fact that small drug dosages were used when compared to those used successfully in the NOD mouse, that the patients were treated after the development of overt diabetes while animals were treated in the pre-diabetic state, and that standardisation of ICA determinations was not indicated in international studies [5]. In particular, of seven high-risk relatives evaluated in the United States, all with initial low first phase insulin secretion, six have developed diabetes. Other reports indicate some efficacy of the drug in pre-diabetic patients, but the studies are without concurrent controls.

The major limitation for such trials is the identification of the 1/150 first degree relalives at increased risk for the development of diabetes. Screening of the general population for Type 1 diabetes risk may be possible in the future using polymerase chain reaction analysis for highrisk HLA alleles (DR3/DR4, DQB1*0302/DQB1*0201). Applying the more detailed analyses used to evaluate first degree relatives of probands as described above on an HLA-selected subgroup of the general population is particularly important in that approximately $80 \%$ of individuals who develop Type 1 diabetes do not have first degree relatives with the disease.

Interpretation of the results of proposed clinical trials must take into account epidaemiologic data indicating that disease onset to a great extent occurs around the time of puberty. Establishment of an international registry of diabetes prevention trials was suggested. Some participants in the workshop suggested that there is a need for the development of non-invasive techniques in which structural and functional islet-cell mass can be assessed and correlated with investigations of insulin secretion.

\section{Treatment of overt Type 1 diabetes at onset}

Early Type 1 diabetes most likely represents part of a continuum of loss of islet-cell function from the prediabetic state to overt disease. Thus, as reviewed by Bach, redefinition of "new onset" diabetes using ICA titres associated with abnormalities of glucose tolerance or insulin secretion as described above may be required.

Immunotherapy with cyclosporin A (CA) and azathioprine in recent-onset Type 1 diabetes can slow the progression of the disease in some patients and may even produce long-term remissions when given within 2 months of the beginning of insulin therapy $[6,7]$. This effect can be obtained with low toxicity if the pharmacokinetics of the drug and renal function are carefully monitored. However, caution regarding the potential long-term oncogenic effects of this agent must be maintained. No increased infection rates have been noted. Preliminary CA treatment trials in recent-onset diabetic patients yielded relatively low $(-30 \%)$ remission rates and showed that relapses could occur while still taking the drug. A possible limitation of these studies was that euglycaemia was not aggressively maintained using exogenous insulin therapy in order to prolong endogenous islet function. Early initiation of insulin therapy as suggested above may allow a larger Beta-cell mass to be preserved and thereby dampen an autoimmune response. It has been proposed that monoclonal T-cell antibodies (anti-T-cell receptor, antiCD3 and anti-CD4) or conjugates of human interleukin 2 with diphtheria toxin may be used to "freeze" the immune response during the time longer acting immunosuppressive agents such as CA are started. These treatments, in conjunction with aggressive control of plasma glucose, may improve the outcome of earlier trials. The ultimate goal is to provide safe and effective immunotherapy of recent-onset diabetes using more selective immunosuppressive agents which could be ultimately used to treat pre-diabetes. 


\section{Risk factors for development of diabetic nephropathy}

Diabetic nephropathy, the most common cause of endstage renal disease in the United States, was reviewed by Seaquist. This complication is associated with increased morbidity and mortality due to macrovascular disease [8]. It is a duration-dependent complication that occurs in a subset $(-30 \%)$ of diabetic patients. Studies in animals as well as epidemiologic data and clinical trials in small numbers of humans suggest a role for the degree of metabolic control in the pathogenesis of kidney complications. Large clinical trials such as the Diabetes Complications and Control Trial may shed more light on the contribution of the metabolic milieu in the development of the devastating complications of this disease.

The development of management schaema for intervention trials of patients at increased risk for diabetic complications requires knowledge of factors which render patients susceptible to the development of nephropathy. Therefore, one of the goals of current research is to develop more sensitive biochemical and genetic markers which will identify patients prior to the onset of irreversible changes in renal structure or function. The potential role of genetic factors in the development of diabetic nephropathy was recently investigated through an examination of familial clustering of subjects with nephropathy [9]. Diabetic siblings of patients with diabetic nephropathy developed nephropathy (albumin excretion rates $>45 \mathrm{mg} / 24 \mathrm{~h}$ ) more frequently $(83 \%)$ than diabetic siblings of diabetic subjects without nephropathy (17\%).

Familial clustering of diabetic nephropathy may be related to the concomitant clustering of hypertension. In some populations, elevated blood pressure was more prevalent in the parents of subjects with diabetic nephropathy than in those without this complication [10]. Elevated erythrocyte cell $\mathrm{Na} / \mathrm{Li}$ countertransport, a marker for essential hypertension, has also been linked to the renal disease of diabetes to an elevation in glomerular filtration rate, an early functional marker of diabetic nephropathy [11]. The physiological significance of $\mathrm{Na} / \mathrm{Li}$ countertransport abnormalities may be related to alterations in the structure or function of $\mathrm{Na} / \mathrm{H}$ antiporters, which modulate intracellular $\mathrm{pH}$ and cell volume. An increase in the $\mathrm{V}_{\max }$ of $\mathrm{Na} / \mathrm{H}$ antiport activity and an increase in the levels of antiporter mRNA in leucocytes of patients with diabetic nephropathy have been reported [12]. Interestingly, Krolewski and co-workers [13] found by logistic regression analysis that the risk conferred by elevated rates of $\mathrm{Na} / \mathrm{Li}$ countertransport was significant only for those individuals with the poorest metabolic control. Thus, at least in this population, glycaemic control may modify the risk determined by one proposed susceptibility factor. It must be emphasised, however, that not all investigators have detected an association between parental hypertension or elevated erythrocyte $\mathrm{Na} / \mathrm{Li}$ countertransport rates and diabetic nephropathy. This suggests that various factors may modify risk of developing diabetic nephropathy to a different extent in different populations.

In an attempt to elucidate genetic factors that render some patients with diabetes susceptible to nephropathy, investigators have searched for links with structural heterogeneity of certain candidate genes. A particular allelic pattern in the $5^{\prime}$ region of the insulin gene has been associated with the development of nephropathy in one patient population [14]. However, efforts to link abnormalities in the renin gene to nephropathy were not successful.

An alternative mechanism whereby the metabolic milieu and the genetic environment may interact in the development of the diabetic nephropathy is in basement membrane metabolism. Patients with diabetic nephropathy were found to have an excess of the 3.6/2.5 genotype of the alpha 1 chain of type IV collagen [15]. These allelic differences may determine heterogeneity in tissue responses to stimulation of extracellular matrix synthesis and thereby the rate of accumulation of glomerular extracellular matrix. Indeed, human umbilical vein endothelial cells from different individuals showed a variation in glucose-induced fibronectin and type IV collagen mRNA levels. Interestingly, these elevated mRNA levels persisted over several cell divisions despite the fact that the cells were returned to media containing normal glucose concentrations [16]. These results suggest that cells may have a "memory" for past metabolic environments, carrying over the consequences of elevated glucose levels to future cell generations. This cell memory may be related to the persistence of advanced glycation end products in nuclear materials or in the extracellular matrix. Thus, the presence of the diabetic state may accentuate genetic differences in extracellular matrix synthesis or in the structure and assembly of its various constituents or both.

\section{Antihypertensive therapy in prevention of diabetic nephropathy}

Microalbuminuria, defined as the absence of detectable proteinuria in association with a persistent albumin excretion rate (AER) between 20 and $200 \mu \mathrm{g} / \mathrm{min}$, has been proposed as a predictor of the development of clinically significant diabetic nephropathy [17]. Its presence heralds a steady fall in the glomerular filtration rate due to expansion of the glomerular extracellular matrix as it encroaches upon the glomerular filtration surface [18]. The description of microalbuminuria as a predictor of the progression towards functional renal impairment in diabetes has led to the institution of clinical trials in animal models of disease as well as in humans [19] designed to prevent deterioration in renal function using antihypertensive agents. 
Antihypertensive therapy prevents diabetic nephropathy in animal models of diabetes. Studies of the efficacy of antihypertensive therapy in prevention of diabetic nephropathy have been performed in the streptozotocininduced (STZ) diabetic rat model $[20,21]$. As reported by Jerums, the former develop the pathologic changes of diabetic nephropathy, including glomerular basement membrane (GBM) thickening and mesangial expansion, without progression to renal failure. While the hormonal environment and the progression of the disease are not identical to those of human diabetes, studies in this model allow for more exploration of structure-function relationships using specific antihypertensive agents which assist in designing more informative studies in humans.

The presence of hypertension accelerated the progression of the diabetic nephropathy. In the hypertensive (SHR)-STZ model, there was a predictable rise in AER starting at 8-12 weeks, so that by 32 weeks there was a greater than 20-fold increase in AER. By comparison, hypertension alone or diabetes alone were associated with a three- to six-fold increase in AER. The SHR-STZ rat also showed a 30-50\% increase in GBM thickness and glomerular volume and similar changes in fractional mesangial volume.

Treatment with angiotensin converting enzyme (ACE) inhibitors decreases angiotensin II generation while activating the renal kallikrein-kinin-prostaglandin system. It lowers both systemic arterial as well as intraglomerular pressures, preventing the development of albuminuria in hypertensive SHR or non-hypertensive STZ diabetic rats. Whether or not ACE inhibition is a specific therapy for the diabetic nephropathy or whether lowering of blood pressure in the presence or absence of an activated renin angiotensin system is as effective remains controversial.

Enalapril has been compared with hydralazine/ metoprolol, and perindopril has been compared with triple therapy consisting of hydrochlorothiazide, reserpine, and hydralazine. All of the treatment regimens lowered the systemic blood pressure to equal degrees and prevented the rise in AER and GBM thickening to a similar degree in the STZ and SHR-STZ diabetic rat models [20]. Fractional mesangial volume was not influenced by any antihypertensive regimen. In the normotensive SpragueDawley rat, similar results were obtained for the AER, which showed minimal increases over 32 weeks in rats treated with perindopril or triple therapy. However, in contrast to the SHR-STZ model, the effects on AER were not accompanied by any amelioration of glomerular ultrastructural changes.

Preliminary studies have been performed in the SHRSTZ rat in an effort to define organ-specific actions of ACE inhibitors in diabetic microvascular disease. Using the double isotope albumin permeability technique introduced by Williamson and co-workers, Jerums and his group showed that the three-fold increase in retinal albumin permeability present in SHR diabetic rats was prevented by antihypertensive therapy with ACE inhibitors, triple therapy, or using calcium channel blockers. The latter treatment was most effective in restoring normal albumin permeability.

In summary, the studies presented at this workshop have not demonstrated a specific benefit of ACE inhibition over the effects of other antihypertensive regimens in experimental diabetic nephropathy. Future animal studies employing new techniques for organ-specific assessment of vascular permeability in combination with structurefunction and biochemical studies will be necessary to determine the relative benefits of each of the proposed antihypertensive regimens.

Antihypertensive therapy prevents diabetic nephropathy in human diabetes [19]. The Melbourne Diabetic Nephropathy Study Group, reviewed by Jerums, has undertaken studies in humans to compare the effects of ACE inhibition using perindopril with the effects of calcium channel blockade using nifedipine in microalbuminuric Type 1 and Type 2 diabetic patients. Normotensive $(n=30)$ and hypertensive $(n=13)$ subjects were started on this regimen. There was a fall in AER in the hypertensive patients and a stabilisation of albumin excretion in the normotensive group over 12 months. In 10 patients for whom 3 years of prior AERs were available, antihypertensive therapy interrupted a progressive rise in AER. No difference in the two modes of antihypertensive therapy was detected. There was also a significant correlation between changes in blood pressure and AER in individual patients. No change in glomerular filtration rate was detected over the 12-month study period. One month after cessation of therapy, there was a rise in blood pressure of $15 \mathrm{~mm} \mathrm{Hg}$ and a rise in AER in both treatment groups. A placebo-controlled, randomised extension of this study in normotensive microalbuminuric patients which will include structural as well as functional renal studies is in progress. Use of plasma prorenin measurements may help to identify patients with early microvascular disease.

Mogensen proposed that the natural history of diabetic nephropathy begins with glomerular hyperfiltration and takes on average 30 years to progress through microalbuminuria and proteinuria to end-stage renal failure. Studies of the effects of various therapies must use end points other than renal failure, focussing on a predictable, progressive fall in glomerular filtration rates. The level of microalbuminuria may also be useful in these studies, but its level may vary somewhat, emphasising the need for reproducible urinary collection techniques. Comparison of enalapril and metoprolol, both given with frusemide, in patients with diabetic nephropathy showed that the former treatment resulted in a slower fall in glomerular filtration rate $\left(2 \mathrm{ml} \cdot \mathrm{min}^{-1} \cdot\right.$ year $\left.^{-1}\right)$ when compared with diabetic patients with albuminuria who were untreated $\left(10 \mathrm{ml} \cdot \mathrm{min}^{-1} \cdot \mathrm{year}^{-1}\right)$. Prospective analysis of the effect of ACE inhibitors in normotensive 
microalbuminuric patients also showed a protective effect of this treatment on the development of overt proteinuria. In this study, none of the 21 patients treated with captopril developed proteinuria, while seven of 23 untreated patients developed proteinuria associated with a fall in the glomerular filtration rate. Whether or not the effects of this treatment were independent of changes in blood pressure is disputed and emphasises the necessity of blinded placebo controlled trials. Indeed, in another study $50 \%$ of the reduction in AER was attributed to reduction in blood pressure (Mogensen CE, unpublished observation). Diabetes did not alter the diurnal variability of blood pressure, but blood pressure was elevated at all times during the day in these subjects.

Future studies will require more detailed examination of the effects of diabetic nephropathy and antihypertensive therapy on the normal diurnal variation in blood pressure and glomerular haemodynamics. Correlation of these physiological measurements with measurements of the glomerular extracellular matrix area will be helpful in sorting out the importance of various treatment modalities in reversal or amelioration of the progressive diminution of glomerular filtration surfaces. Whether good dietary control of the disease and its metabolic consequences, such as dyslipidaemia and hyperglycaemia, impact independently on the progression of diabetic renal disease needs further study in both animal and human diabetes.

Pancreas transplantation: metabolic consequences and associated effects on chronic complications of diabetes

Pancreas transplantation, reviewed by Robertson, was performed in over 700 patients worldwide in 1990, with more than 3000 diabetic patients having received this mode of treatment to date $[22,23]$. Graft survival in the United States is currently approximately $60 \%$, with approximately $90 \%$ patient survival rate 3 years after transplantation. Work in this field has yielded important new information regarding the natural history and pathophysiology of Type 1 diabetes. For example, from twin-to-twin transplantation, we know that the immune system retains memory capable of repeating autoimmune destruction of islets up to 17 years after the initial diabetic insulitis [24].

Fasting glucose, intravenous glucose disposal, and haemoglobin $A_{1 C}$ can be normalised for many years following pancreas transplantation [25]. Insulin levels in both the basal and stimulated state are increased due to the necessity for systemic pancreatic venous drainage of the grafted organ [26] which avoids first pass hepatic degradation of insulin. Glucocorticoid therapy is included in immunosuppressive regimens and is another factor resulting in hyperinsulinism because it causes insulin resistance. Recovery from insulin-induced hypoglycaemia is improved in allograft recipients, in part due to improved glucagon secretion. On the other hand, pancreatic polypeptide responses to hypoglycaemia do not improve, reflecting the absence of pancreatic innervation of the allograft.

While correction of the metabolic consequences of diabetes is impressive, whether or not this treatment prevents or reverses the chronic complications of diabetes has been difficult to ascertain. Since the usual recipient of a transplanted pancreas has had diabetes for over 20 years, it may not be reasonable to expect normalisation of glucose levels to reverse or even stabilise chronic complications. Studies have not shown an improvement in diabetic retinopathy after achievement of normoglycaemia in pancreas recipients [27], but the presence of a transplanted pancreas appears to protect the kidney from further damage [28]. Interpretation of kidney results is complicated by cyclosporin which affects renal function. Motor and sensory nerve conduction improve with pancreatic transplantation [29]. Overall patient survival also improves in patients with autonomic nervous system dysfunction as reflected by abnormal cardiorespiratory reflex responses [30]. Quality of life studies, although few in number, seem to indicate that patients who have received a successful pancreas transplant feel that they have improved lives.

It must be cautioned, however, that all studies to date have been performed in a non-randomised fashion in selfselected patients, most of whom had already developed chronic complications of diabetes. In the future, appropriately designed randomised prospective trials, performed in patients believed to be at increased risk for the complications of diabetes, or who are in an early stage of these complications, need to be considered.

\section{Pancreatic islet transplantation for Type 1 diabetes}

Pancreatic islet autografts, reviewed by Mintz, are successful in large mammals, including humans, with pancreatectomy-induced diabetes [31]. The number of autografted islets required is considerably lower than is allografted islets. Islets from multiple donors have been required to achieve normal intravenous glucose tolerance with pancreatectomy-induced diabetes or in spontaneous diabetes [32].

Designing strategies to achieve successful islet transplants in man has therefore either required the availability of cryopreserved islets, or successive fresh donor pancreases, or both. There has been only one unique clinical situation that is an exception to this statement. In patients undergoing upper abdominal exenterations for advanced malignancies and who also had concurrent combined liver-islet allografts, single donated pancreases yielded sufficient islets to prevent insulin-dependent diabetes as a consequence of the pancreatectomy in this "cluster" operation. This provided unequivocal evidence that islet transplants can function long term in man [33] (now 3 years in the longest surviving patients) and has catalysed considerable interest in islet transplantation in patients with long-standing 
Type 1 diabetes. These recipients either have had stable kidney allografts or were about to receive a new kidney allograft. Islets were harvested from more than one pancreas donor (either freshly isolated, or cryopreserved, or both). Typically, cryopreserved islets from multiple donors or sequentially isolated islet preparations from two to four donors were used.

The results of these new trials are cause for both optimism and the need for continued study. On the positive side, insulin independence has been achieved for variable lengths of time in patients at the University of Alberta, Washington University, the University of Milan and the University of Miami. However, only one of the several dozens of patients studied to date has been insulinindependent for a prolonged period (greater than 2 years) [34]. In the Miami experience, the continuing use of lowdose insulin has been associated with a high level of persistent graft function in the last three consecutive patients. Each recipient has had a functioning islet allograft which has lasted for more than 1 year.

The mechanisms underlying islet allograft failure in these trials has been somewhat clarified. Allograft rejections seem to be the primary cause of graft failure. In Miami, three of six patients have been shown to develop cytotoxic antibodies against each donor's lymphocytes (frozen). These antibodies appeared prior to graft failure and were therefore highly suggestive of incipient allograft rejection. In addition, islet graft failure can be related to the quality of the islets that were transplanted, or the quantity of islets that were engrafted, or both.

In a comparative study between laboratories in Pittsburgh (C. Ricordi) and Miami (R. Alejandro), $78 \%$ of islet preparations obtained in Pittsburgh were deemed acceptable for transplantation whereas only $56 \%$ were acceptable in Miami. These results highlight that a myriad of uncontrollable donor factors affect the quality and function of isolated islets. The potentially noxious conditions to which islets are exposed include anoxia, drugs administered during donor life support, excessive intrapancreatic pressure during hypothermic perfusion (unless the pancreatic venous system is vented), warm ischaemic damage, hyperosmolarity and other physical damage to islets during their isolation, and loss of viability of islets in vitro through freeze-thaw damage. Although the methods for quantification are improved, the conditions surrounding this enumeration are not standardised, and comparison of islet transplantable mass between centres is still not possible. Lastly, the liver may not be the ideal site for engraftment. Intrahepatic islets are downstream from post-prandial portal vein surges of hyperglycaemia which raises the possibility of islet glucotoxic effects. This may account for the seemingly better long-term results of intrasplenic autografts in experimental animals. Whether continuous low-dose insulin therapy protects islet allografts is still unknown, although the prolonged survival of islet allograft function in the Miami series suggests that this may be the case.
The future directions for islet transplantation will be based upon current trials of human islet allografts. However, more research is needed to achieve a substantially greater bulk of islets for transplantation. This is unlikely to come from human sources. Thus, research in the use of islet xenografts, and bio-engineered Beta cells should be expanded. Moreover, efforts to understand the mechanisms underlying tolerance induction for islet alloand xenografts, or for the immunoprotection of islet transplants, should be given a high priority for increased funding.

\section{The insulin-like effects of vanadate}

Over the past decade, as discussed by Shechter, it has been demonstrated that vanadate ions, which inhibit phosphohydrolases in the $\mu \mathrm{mol} / 1-\mathrm{mol} / 1 \mathrm{range}$, mimic physiological actions of insulin [35]. These actions persist even after the down regulation of insulin receptors, without altering insulin production or receptor binding. This includes stimulation of glucose uptake and metabolism, activation of glycogen synthase, and inhibition of lipolysis. The ion also stimulates potassium uptake and calcium influx and decreases the intracellular $\mathrm{pH}$. Indeed, vanadate even mimics insulin action at the level of the genome by increasing L-type pyruvate kinase gene expression in primary adult rat hepatocyte cultures [36]. Interest in vanadate escalated when it was shown that oral administration of the ion lowered blood glucose levels in STZ-treated diabetic rats [36-39]. Oral vanadate also restored levels of glycogen and fructose-2,6 bisphosphate kinase in the liver, glucose transporters in muscle and improved tissue responsiveness to insulin. Oral vanadate therapy also induced normoglycaemia in highly insulinresistant rodents such as $\mathrm{db} / \mathrm{db}$ and ob/ob mice [40]. Taken together, these in vitro and in vivo findings indicate that vanadium salts or a related compound may play a role in the future treatment of diabetes.

With respect to the site of action of vanadate, recent information has indicated that the $40,000 \times g$ supernatant fraction from broken rat adipocyte homogenates contained a soluble tyrosine kinase. This newly discovered enzyme was activated by vanadate and was distinct from the insulin-receptor kinase since it was cytosolic and had an estimated molecular weight of $53 \mathrm{kDa}$. In addition, its activity was supported by $\mathrm{Co}^{2+}$ rather than $\mathrm{Mn}^{2+}$, while the insulin receptor is $\mathrm{Mg}^{2+}$-dependent. The enzyme was activated by vanadate ions and inhibited by staurosporine $\left(\mathrm{IC}_{50}=20-2 \mathrm{nmol} / \mathrm{l}\right)$. Several physiological lines of evidence strongly suggest that this tyrosine kinase is the site of action of vanadate ions in mediating its insulin-like biological effects. 
The sulphonylurea receptor and Beta-cell signal transduction

One of the primary defects associated with Type 2 diabetes is an inability of the Beta cell to respond appropriately to the primary physiological secretagogue for insulin, glucose. Since the 1950s, sulphonylureas have been used to treat patients with this disease. The progress in understanding how both glucose and sulphonylureas act on the Beta cell to trigger insulin release was discussed by Boyd. Beta cells, like neurons or myocytes, are electrically active cells. On exposure to glucose or sulphonylureas, there is an increase in Beta-cell electrical activity which causes insulin release. Both agents act on the same ion channel in the Beta cell to initiate a cascade of events leading to insulin exocytosis. Glucose metabolism increases the ATP/ADP ratio and inhibits the ATPsensitive $\mathrm{K}^{+}$channel, leading to intracellular $\mathrm{K}^{+}$retention and depolarisation. The change in membrane potential opens the voltage-dependent calcium channels (VDCC) and allows extracellular $\mathrm{Ca}^{2+}$ to flow down the 10,000 -fold concentration gradient into the cell. The rise in free cytosolic calcium $\left[\mathrm{Ca}^{2+}\right]$ i triggers insulin release.

Sulphonylureas bind with high affinity to a receptor on the Beta-cell surface which may be the ATP-sensitive $\mathrm{K}^{+}$ channel or a closely associated protein [41]. There is a tight correlation between the affinity of these agents for the receptor and inhibition of ${ }^{86} \mathrm{Rb}^{+}$efflux from the ATP sensitive $\mathrm{K}^{+}$channel, increase in $\left[\mathrm{Ca}^{2+}\right] \mathrm{i}$, and increase in insulin secretion. However, sulphonylureas do not increase insulin synthesis. All of these effects occur in the low nmol/l concentration range using the second generation sulphonylureas such as glyburide or glipizide. The sulphonylurea receptor has been solubilised and partially characterised after photoaffinity labelling, but no cDNAs for this receptor have been isolated.

The VDCC is composed of five subunits. The alpha- 2 subunit is capable of forming a pore and passing current. cDNAs to this subunit isolated from hamster $S V 40$ transformed insulinoma or HIT cells revealed that this protein was composed of 1610 amino acids and is in the brain $\mathrm{Ca}^{2+}$-channel gene family. The HIT cell VDCC has a unique carboxyl terminus which was used to identify VDCC mRNAs in pancreas, insulin-secreting cell lines, brain, heart, and skeletal muscle. Using the polymerase chain reaction, primers specific for the HIT cell VDCC were found to have the same widespread distribution. Conversely, a cardiac-like alpha- 2 subunit was also detected in Beta cells. This channel subunit is in the same gene family as that recently cloned from a human insulinoma. However, the latter was much larger than that isolated from HIT cells, extending over 500 amino acids at the carboxyl terminus. The alpha- 2 subunit contains five cyclic AMP-dependent phosphorylation sites on its cytoplasmic loops and three extracellular glycation sites. Strategies can now be developed to increase insulin secretion by directly targeting the VDCC alpha-2 subunit, accepting the necessary precaution that these channels may not be sufficiently cell type specific for selective alterations.

\section{The glucotoxicity hypothesis}

In order to design new approaches to managing Type 2 diabetes and to better understand its pathogenesis, Rossetti reviewed which of the several defects in insulin secretion and insulin action associated with this syndrome are acquired and which are present at the onset of the disease.

One experimental approach was to induce diabetes in 4to 6-week-old Sprague-Dawley rats by $90 \%$ surgical pancreatectomy and to study the relationship between plasma insulin concentrations and intracellular glucose metabolism 5 weeks following the onset of hyperglycaemia $[39,42,43]$. Glucose uptake, whole body glycolysis, muscle glycogen synthesis, and rectus muscle glucose 6phosphate (G-6-P) concentrations were measured in the basal state and during infusion of insulin [42]. The contribution of glycolysis decreased and that of muscle glycogen synthesis increased as the insulin levels rose. Insulin-mediated glucose disposal was decreased by 20 $30 \%$ throughout the insulin dose response curve in diabetic rats compared to control rats. At low insulin infusion rates ( 2 and $3 \mathrm{mU} \cdot \mathrm{kg}^{-1} \cdot \mathrm{min}^{-1}$ ), a reduction in both glycolytic and glycogenic flux contributed to the defective tissue glucose uptake in diabetic rats, while at higher insulin doses impairment in muscle glycogen repletion accounted for the entire difference between diabetic and control rats. Muscle G-6-P concentration was decreased compared to basal at lower insulin infusion rates, but gradually increased twofold as insulin concentrations rose. The G-6-P concentration in diabetic rats was similar to that in controls despite the reduction in glucose uptake. These data suggest that glucose transport is the major determinant of glucose disposal at low insulin concentrations, while the ratelimiting step at high physiological insulin concentrations shifts to an intra-cellular site. Thus, prolonged moderate hyperglycaemia and hypoinsulinaemia result in two distinct cellular defects in skeletal muscle at the level of glucose transport/ phosphorylation and at the level of glycogen synthesis.

The cellular mechanisms responsible for these metabolic defects were further characterised in isolated adipocytes and muscle homogenates from diabetic and control rats. Glucose transport activity as well as GLUT 4 protein and mRNA levels were decreased in isolated adipocytes from diabetic rats when compared to controls. GLUT 4 protein and mRNA levels as well as glycogen synthase $V_{\max }$ were diminished in skeletal muscle from diabetic rats.

The role of chronic hyperglycaemia in mediating secondary defects in glucose-induced insulin secretion [43, 44] and in peripheral insulin action $[39,43,46-48]$ was addressed using phlorizin treatment ( 3 to 4 weeks) in 
diabetic rats. This agent lowers the renal threshold for glucose by inhibiting Na-dependent kidney proximal tubule brush border glucose transport, thereby normalising plasma glucose concentrations without altering the plasma insulin levels. Correction of chronic hyperglycaemia with phlorizin was associated with a reversal of defective glucose-induced insulin secretion in diabetic rats as assessed by hyperglycaemic clamp studies in conscious rats [45]. Interestingly, first phase insulin secretion, which was severely blunted in untreated diabetic rats, was completely normalised by phlorizin. These data suggest that in the presence of a reduced Beta-cell mass, prolonged moderate hyperglycaemia may be responsible for defects in insulin secretion.

The effect of chronic hyperglycaemia on peripheral insulin action was examined using the insulin clamp technique in conscious rats $[39,45,46]$. Phlorizin treatment completely normalised the ability of insulin to stimulate whole body glucose disposal in diabetic rats. However, the ability of insulin to promote in vivo muscle glycogen synthesis and to activate skeletal muscle glycogen synthase remained markedly impaired [39]. Thus, the reversal of chronic hyperglycaemia appears to selectively correct the impairment in insulin action on muscle glucose uptake but not on glycogen synthesis.

To investigate the cellular and molecular mechanism(s) responsible for the effect of chronic hyperglycaemia on peripheral insulin action, isolated adipocytes obtained from control, diabetic and diabetic phlorizin-treated rats were examined [46]. Insulin-stimulated glucose transport activity was decreased by $50 \%$ and was completely normalised following the correction of chronic hyperglycaemia with phlorizin treatment, consistent with the insulin-mediated glucose uptake in vivo. However, following phlorizin treatment, levels of GLUT 4 protein remained reduced by $46 \%$ in the plasma membrane in the insulin-stimulated state and GLUT 4 mRNA levels remained depressed by approximately $50 \%$. Thus, correction of chronic hyperglycaemia normalises peripheral glucose uptake in vivo and glucose transport activity in isolated adipocytes in the absence of any change in glucose transporter number or its mRNA. Since this condition is associated with changes in insulin stimulation of glucose uptake in vivo, it raises the question of whether or not GLUT 4 expression is the major determinant of in vivo glucose uptake.

In summary, the observations outlined herein emphasise the complexity and heterogeneity of the metabolic/molecular alterations unified under the name "insulin resistance." These results suggest that chronic hyperglycaemia may promote defective insulin action on glucose transport in Type 1 and Type 2 diabetes and can occur in the absence of any detectable change in glucose transporter expression. On the contrary, other intracellular defect(s) in insulin action, e.g., impaired activation of muscle glycogen synthase, are not inducible by chronic hyperglycaemia.

\section{Conclusions and recommendations}

Laboratory measures are being developed which will be employed to more precisely predict the development of Type 1 diabetes and its chronic complications. These measures can be utilised in human and animal studies whose aim is reversal or prevention of islet cell destruction. There is a need for more specific immunomodulating agents to be used in the treatment of diabetics with residual islet cell function. A more precise understanding of the genetic locus or loci primarily responsible for diabetes as well as how environmental factors impact upon disease progression will help researchers design more specific therapies to include both pancreas and islet transplantation.

Newer therapies can be designed once there is understanding of the mechanisms of insulin action and of which biochemical and physiological abnormality in Type 1 and Type 2 diabetes is primary to the disease aetiology. Reversal of the metabolic consequences of the disease using pancreatic or islet transplantation or newer insulinomimetic agents will be useful in preventing or halting the progression of the chronic complications of diabetes.

Acknowledgements. This report is the result of Work Group 2: Treatment of Diabetes Mellitus at the Third JDF World Conference on Diabetes in Monte Carlo, Monaco, 8-10 March 1992. Chairman: R. P. Robertson. Work group participants: J.-F. Bach ${ }^{1}$, A. E. Boyd ${ }^{2}$, G. S. Eisenbarth ${ }^{3}$, G. Jerums $^{4}$, D. H. Mintz ${ }^{5}$, C. E. Mogensen ${ }^{6}$, L. Rossetti ${ }^{7}$, E. R. Seaquist ${ }^{8}$, Y. Shechter ${ }^{9}$. Rapporteur: D. J. Klein. Discussants: E. Colle ${ }^{10}$ O. B. Crofford $^{11}$, D. Elias ${ }^{12}$.

${ }^{1}$ Necker Hospital, Paris, France; ${ }^{2}$ Endocrine Division, New England Medical Center, Boston, massachusetts, USA; ${ }^{3}$ Joslin Diabetes Center, Boston, Massachusettes, USA; ${ }^{4}$ Endocrine Unit, Department of Medicine, Austin Hospital, Heidelberg, Victoria, Australia; ${ }^{5}$ Medical School, University of Miami, Miami, Florida; ${ }^{6}$ Diabetes and Endocrinology Department, University Hospital, Aarhus, Denmark; ${ }^{7}$ Division of Endocrinology, Albert Einstein College of Medicine, Bronx, New York, USA; ${ }^{8}$ Diabetes Center, University of Minnesota, Minnesota, Minneapolis, Minnesota, USA; ${ }^{9}$ Department of Hormone Research, Weizmann Institute of Science, Rehovot, Israel; ${ }^{10}$ Montreal Children's Hospital, Montreal, Quebec, Canada; ${ }^{11}$ Diabetes Research and Training Center, Vanderbilt University, Nashville, Tennessee, USA; ${ }^{12}$ Department of Cell Biology, Weizmann Institute of Science, Rehovot, Israel.

\section{References}

1. Castano L, Eisenbarth, GS (1990) Type 1 diabetes: a chronic autoimmune disease of man, mouse and rat. Annu Rev Immunol 8: 647-679

2. Vardi P, Crisa L, Jackson RA et al. (1991) Predictive value of intravenous glucose tolerance test insulin secretion less than or greater than the first percentile in islet cell antibody positive relatives of type 1 (insulin-dependent) diabetic patients. Diabetologia 34: 93-102

3. Baekkeskov S, Warnock G, Christie M, Rajotte RW, Larsen PM, Fey S (1989) Revelation of 64K autoantibodies in IDDM serums by high resolution 2-D gel electrophoresis: unambiguous identification of $64 \mathrm{~K}$ target antigen. Diabetes 38: 1133-1141

4. Gotfredsen CF, Buschard K, Frandsen EK (1985) Reduction of diabetes incidence of BB Wistar rats by early prophylactic insulin treatment of diabetes-prone animals. Diabetologia 28: 933-935 
5. Chase HP, Butler-Simon N, Garg S, McDuffie M, Hoops SL, O'Brien D (1990) A trial of nicotinamide in newly diagnosed patients with type 1 (insulin-dependent) diabetes mellitus. Diabetologia 33: 444-446

6. Stiller CR, Dupre J, Gent $M$ et al. (1984) Effects of cyclosporine immunosuppression in insulin-dependent diabetes mellitus of recent onset. Science 223:1362-1367

7. Bougneres PF, Carel JC, Castano C et al. (1988) Factors associated with early remission of type 1 diabetes in children treated with cyclosporine. N Engl J Med 318: 663-670

8. Borch-Johnsen K, Andersen PK, Deckert T (1985) The effect of proteinuria on relative mortality in type 1 (insulin-dependent) diabetes mellitus. Diabetologia 28: 590-596

9. Seaquist ER, Goetz FC, Rich S, Barbosa JJ (1989) Familial clustering of diabetic kidney disease. N Engl J Med 320: 1161-1165

10. Viberti GC, Keen H, Wiseman MJ (1987) Raised arterial pressure in parents of proteinuric insulin dependent diabetics. Brit Med J 295: 515-517

11. Carr $\mathrm{S}$, Mbanya JC, Thomas $\mathrm{T}$ et al. (1990) lncrease in glo-merular filtration rate in patients with insulin-dependent diabetes and elevated erythrocyte sodium-lithium countertransport. N Engl J Med 322: 500-505

12. Ng LL, Simmons D, Frighi V, Garrido MC, Bomford J, Hockaday TDR (1990) Leucocyte $\mathrm{Na} / \mathrm{H}+$ antiport activity in type 1 (insulindependent) diabetic patients with nephropathy. Diabetologia 33: $371-377$

13. Krolewski AS, Canessa M, Warram JH et al. (1988) Predisposition to hypertension and susceptibility to renal disease in insulindependent diabetes mellitus. N Engl J Med 318: 140-145

14. Raffel LJ, Vadheim CM, Roth M-P, Klein R, Moss SE, Rotter JI (1991) The 5' insulin gene polymorphism and the genetics of vascular complications in type 1 (insulin-dependent) diabetes mellitus. Diabetologia 34:680-683

15. Krolewski A, Tryggvason K, Stanton V, Housman D (1991) Diabetic nephropathy and polymorphism in cDNA of the alpha 1 chain of type IV collagen. J Am Soc Nephrol 2:634

16. Roy S, Sala R, Cagliero E, Lorenzi M (1990) Overexpression of fibronectin induced by diabetes or high glucose: phenomenon with a memory. Proc Natl Acad Sci USA 87:404-408

17. Viberti GC, Hill RD, Jarrett RJ, Argyropoulos A, Mahmud Y, Keen $H$ (1982) Microalbuminuria as a predictor of clinical nephropathy in insulin-dependent diabetes mellitus. Lancet I: 1430-1432

18. Mauer SM, Steffes MW, Enis EN, Sutherland DER, Brown DM, Goetz FC (1984) Structural-functional relationships in diabetic nephropathy. J Clin Invest 74: 1143-1155

19. Mogensen CE (1992) Angiotensin converting enzyme inhibitors and diabetic nephropathy. Brit Med J 304: 327-328

20. Cooper ME, Allen TJ, O'Brien RC et al. (1990) Nephropathy in model combining genetic hypertension with experimental diabetes: enalapril versus hydralazine and metoprolol therapy. Diabetes 39: 1575-1579

21. Zatz R, Dunn BR, Meyer TW, Anderson S, Rennke HG, Brenner BM (1986) Prevention of diabetic glomerulopathy by pharmacological amelioration of glomerular capillary hypertension. J Clin Invest $77:$ 1925-1930

22. Sutherland DER, Gruessner A, Mondry-Munns K (1992) Report on results of pancreas transplantation in the United States October 1987 to October 1991 from the United Network for Organ Sharing Registry. In: Terasaki PI (ed) Clinical transplants. UCLA Tissue Typing Laboratory, Los Angeles, pp 31-38

23. Robertson RP (1991) Pancreas transplantation in humans with diabetes mellitus. Diabetes 40: 1085-1089

24. Sibley, RK, Sutherland DER, Goetz F, Michael AF (1985) Recurrent diabetes mellitus in the pancreas iso- and allograft. Lab Invest 53: 132-144

25. Diem P, Redmon JB, Abid $M$ et al. (1990) Glucagon, catecholamine and pancreatic polypeptide secretion in type 1 diabetic recipients of pancreas allografts. J Clin Invest 86: 2008-2013
26. Diem P, Abid M, Redmon JB, Sutherland DER, Robertson RP (1990) Systemic venous drainage of pancreas allografts as independent cause of hyperinsulinemia in type 1 diabetic recipients. Diabetes 39: 534-540

27. Ramsay RC, Goetz FC, Sutherland DER et al. (1988) Progression of diabetic retinopathy after pancreas transplantation for insulindependent diabetes mellitus. $N$ Engl J Med 318: 208-214

28. Bilous RW, Mauer SM, Sutherland DER, Najarian JS, Goetz FC, Steffes MW (1989) The effects of pancreas transplantation on the glomerular structure of renal allografts in patients with insulindependent diabetes. $N$ Engl J Med 321: 80-85

29. Kennedy WR, Navarro X, Goetz FC, Sutherland DER, Najarian JS (1990) Effects of pancreatic transplantation on diabetic neuropathy. $N$ Engl J Med 322: 1031-1037

30. Navarro X, Kennedy WR, Loewenson RB, Sutherland DER (1990) Influence of pancreas transplantation on cardiorespiratory reflexes, nerve conduction, and mortality in diabetes mellitus. Diabetes 39 : 802-806

31. Farney AC, Najarian IS, Nakhleh RE et al. (1991) Autotransplant of dispersed pancreatic islet tissue combined with total or near total pancreatectomy for treatment of chronic pancreatitis. Surgery 110: $427-437$

32. Alejandro R, Latif Z, Polonsky KS, Shienvold FL, Civantos F, Mintz DH (1988) Natural history of multiple intrahepatic canine islet allografts during and following administration of cyclosporine. Transplantation 45: 1036-1041

33. Ricordi C, Tzakis AG, Carroll PB (1992) Human islet isolation and allotransplantation in 22 consecutive cases. Transplantation 53: 407 414

34. Warnock GL, Kneteman NM, Ryan EA, Rabinovitch A, Rajotte RV (1992) Long-term follow-up after transplantation of insulinproducing pancreatic islets into patients with type 1 (insulindependent) diabetes mellitus. Diabetologia 35: 89-95

35. Shechter $Y(1990)$ Perspective in diabetes: insulin-mimetic effects of vanadate; possible implications for future treatment of diabetes. Diabetes 39: 1-5

36. Helliger CE, Tahiliani AG, McNeill JH (1985) Effect of vanadate on elevated blood glucose and depressed cardiac performance of diabetic rats. Science 227: 1474-1476

37. Gil J, Mirapelx M, Carreras J, Bartrons R (1988) Insulin-like effects of vanadate on glucokinase activity and fructose 2,6- bisphosphate levels in the liver of diabetic rats. J Biol Chem 263: 1868-1871

38. Strout HV, Vacario PP, Biswas C et al. (1990) Vanadate treatment of streptozotocin diabetic rats restores expression of the insulinresponsive glucose transporter in skeletal muscle. Endocrinology 126: $2728-2732$

39. Rossetti L, Laughlin MR (1989) Correction of chronic hyperglycaemia with vanadate, but not with phlorizin, normalises in vivo glycogen repletion and in vitro glycogen synhase activity in diabetic skeletal muscle. J Clin Invest 84: 892-899

40. Meyerovitch J, Rothenberg P, Shechter Y, Bonner-Weir SA, Kahn CR (1991) Vanadate normalises hyperglycaemia in two mouse models of non-insulin-dependent diabetes mellitus. J Clin Invest 87: 1286-1294

41. Boyd AEI (1988) Sulfonylurea receptors, ion channels, and fruit flies. Diabetes 37: 847-850

42. Rossetti L, Giaccair A (1990) Relative contribution of glycogen synthesis and glycolysis to insulin-mediated glucose uptake: a doseresponse euglycaemic clamp study in normal and diabetic rats. $J$ Clin Invest 85; 1785-1792

43. Rossett L, Shulman G1, Zawalich W, DeFronzo RA (1987) Effect of chronic hyperglycaemia on in vivo insulin secretion in partially pancreatectomised rats. I Clin Invest 80: 1037-1044

44. Leahy JL, Bonner-Weir S, Weir GC (1988) Minimal chronic hyperglycaemia is a critical determinant of impared insulin secretion after incomplete pancreatectomy. J Clin Invest 81: 1407 1414

45. Rossetti L, Smith D, Shulman GI, Papachristou D, DeFronzo RA 
(1987) Correction of hyperglycaemia with phlorizin normalises tissue sensitivity to insulin in diabetic rats. J Clin Invest 79: 15101515

46. Kahn BB, Shulman GI, DeFronzo RA, Cushman SW, Rossetti L (1991) Normalisation of blood glucose in diabetic rats with phlorizin treatment reverses insulin-resistant glucose transport in adipose cells without restoring glucose transporter gene expression. J Clin Invest 87: $561-570$
47. Richter EA, Hansen BF, Hansen SA (1988) Glucose-induced insulin resistance of skeletal muscle glucose transport and uptake. Biochem J 252: 733-737

48. Garvey WT, Olefsky JM, Matthaei S, Marshall S (1987) Glucose and insulin co-regulate the glucose transport system in primary cultured adipocytes. J Biol Chem 262: 189-197 